\title{
ULTRAVIOLET TO NEAR-INFRARED SPECTRAL DISTRIBUTIONS OF STAR-FORMING GALAXIES: METALLICITY AND AGE EFFECTS
}

\author{
Thaisa Storchi-BergmanN ${ }^{1}$ \\ Departamento de Astronomia, IF-UFRGS, CP 15051, CEP 91501-970, Porto Alegre, RS, Brasil \\ AND \\ Daniela Calzetti ${ }^{2}$ and AnNe L. Kinney ${ }^{1,2}$ \\ Space Telescope Science Institute, 3700 San Martin Drive, Baltimore, MD 21218 \\ Received 1993 September 13; accepted 1993 December 7
}

\begin{abstract}
Spectral distributions from the UV to the near-IR of a sample of 44 star-forming galaxies are used to calculate the metallicity $(\mathrm{O} / \mathrm{H})$, star-formation rate (SFR) and age of the starbursts. The oxygen abundance covers the range $8.3<\mathrm{O} / \mathrm{H}<9.4$, and nitrogen $(\mathrm{N})$ is found to be mostly a product of secondary nucleosynthesis for $\mathrm{O} / \mathrm{H}>$ 8.4. Due to its secondary origin, $\mathrm{N} / \mathrm{O}$ ratios up to $\approx 4$ times the solar value can be obtained for metalrich starbursts. The SFR ranges from 0.01 to $100 \mathrm{M}_{\odot} \mathrm{yr}^{-1}$. The lower metallicity galaxies seem to be experiencing an instantaneous burst of star formation, with ages ranging from under $5 \times 10^{6}$ to $10^{7} \mathrm{yr}$. The highest metallicity galaxies are most probably experiencing a continuous burst.

Correlations between the calculated quantities and several spectral features are investigated. We found a highly significant correlation between the equivalent width $W(\mathrm{C}$ IV $\lambda 1550$ ) - a stellar (absorption) feature-and the oxygen abundance of the emitting gas $(\mathrm{O} / \mathrm{H})$. Thus we show for the first time that the stellar metallicity is well correlated with the gas metallicity in star-bursting galaxies. The equivalent width $W(\mathrm{Si}$ IV 21400$)$ and the emission line ratio [N II] $\lambda \lambda 6548,84 / \mathrm{H} \alpha$ also correlate well with $\mathrm{O} / \mathrm{H}$, and all three features can be used as metallicity indicators for star-forming galaxies.

The continuum color between $\lambda 1400$ and $\lambda 3500$ [C(14-35)] is shown to correlate with $\mathrm{O} / \mathrm{H}$, although it is better correlated with $E(B-V)$. It was not possible to disentangle the metallicity from the reddening effect in $C(14-35)$. We estimate that the reddening affecting the UV continuum is about half the one derived from the Balmer decrement of the emitting gas.

The SFR correlates well with the galaxy luminosity and there is no dependence of the continuum color on the SFR.

The higher metallicities are only found in the more luminous galaxies, while low metallicities are found over the whole luminosity interval $\left(-16<M_{B}<-23, H_{0}=50 \mathrm{~km} \mathrm{~s}^{-1} \mathrm{Mpc}^{-1}\right)$ covered by the sample.

Subject headings: galaxies: abundances - galaxies: evolution - galaxies: photometry - galaxies: starburst
\end{abstract}

\section{INTRODUCTION}

Star-formation phenomena are among the most fundamental processes studied in astrophysics. The investigation of the characteristics and nature of the star-formation, its dependence on mass and metallicity are essential to understand the evolution of stellar populations in galaxies, and can give clues on the nature of distant galaxies. Indeed, recent photometric and spectroscopic data on galaxies of intermediate redshift $(0.5 \leq z \leq 1)$ shows an excess of blue galaxies relative to nearby samples (e.g., Ellis 1992, and references therein; Dressler \& Gunn 1983; Dressler, Gunn, \& Schneider 1985), indicating higher frequency of star-formation processes in the past. Also, due to the effects of $k$-corrections and cosmological surface-brightness dimming, star-forming galaxies should be preferentially selected in surveys of high-redshift galaxies (e.g., Tyson, 1984, 1988). Knowledge of the star-formation rate (SFR) throughout the universe as a function of space and time is the primary goal of both observers and theorists who work

\footnotetext{
${ }^{1}$ Visiting Astronomer at the Cerro Tololo Inter-American Observatory, operated by the Association of Universities for Research in Astronomy, Inc., under contract with the National Science Foundation.

${ }_{2}$ Visiting Astronomer at Kitt Peak National Observatory, operated by the Association of Universities for Research in Astronomy, Inc., under contract with the National Science Foundation.
}

on the area of galaxy formation. For example, the rate of transformation of gas into stars, as a galaxy ages, is a crucial determination of the spectrophotometric properties of that galaxy, and of its Hubble type (Bruzual 1983; Sandage 1986; Guiderdoni \& Rocca-Volmerange 1987).

Recent works also point toward a relation between star formation and nuclear activity (e.g., Terlevich 1990; Terlevich et al. 1992); the high nitrogen abundances found in the narrowline region of Seyfert 2 and LINER galaxies by StorchiBergmann \& Pastoriza (1989, 1990; see also Storchi-Bergmann 1991) and by Hamman \& Ferland (1992) in the UV spectra of QSOs imply that even these objects are probably associated with vigorous star formation.

With the aim of studying the star-formation and its relation to nuclear activity in galaxies we have obtained spectral distributions from $\lambda 1200$ to $\lambda 10000$ for star-forming and Seyfert galaxies. The UV spectral distributions were obtained from the Atlas of Star-Forming Galaxies of Kinney et al. (1993). Optical and near-IR spectra with apertures matching those of the IUE were obtained at the Cerro Tololo Inter-American Observatory and Kitt Peak National Observatory, and are respectively described in Storchi-Bergmann et al. (1994, hereafter Paper I) and in McQuade, Kinney, \& Calzetti (1994, hereafter Paper II). As our data include UV spectroscopy and was obtained through a relatively large aperture $\left(10^{\prime \prime} \times 20^{\prime \prime}\right)$, it is particularly suitable to comparison with data on high-redshift galaxies 
$(z \geq 1)$, for which integrated populations are observed and optical observations correspond to the UV range in the rest frame of the galaxies.

In this paper, we analyse the spectral distributions for the subsample of 44 star-forming galaxies, which includes Starburst, Blue Compact, and $\mathrm{H}$ II galaxies, in order to study the dependence of the spectral characteristics on the metallicity, age, and star-formation rate. In a forthcoming paper (Calzetti, Kinney, and Storchi-Bergmann 1994), the effects of reddening are investigated.

We use the measurements provided in Papers I and II to calculate the physical properties and chemical abundances for the emitting gas, the star-forming rate, and to estimate the age of the last burst of star formation. The relation between these properties and several spectral features like emission-line ratios, absorption and emission equivalent widths, and continuum color are then investigated.

\section{THE SAMPLE}

The sample comprises six Blue Compact Dwarf Galaxies (BCDG), eight Blue Compact Galaxies (BCG), 17 Starbursts
(SB), six H II galaxies (H II), two Hot Spot galaxies, four unclassified and one Composite (Seyfert + Starburst) but where the starburst characteristics are clearly dominant. Spectral distributions for the galaxies are shown in Papers I and II, as well as measurements for the fluxes in several points of the continuum and in all the emission lines. Equivalent widths of the strongest absorption features were also measured and presented in Paper I. The sample galaxies are identified in the first column of Table 1, which also lists the properties calculated in the following sections.

\section{GAS PROPERTIES}

\subsection{Reddening}

In order to calculate the gas temperature, density, and chemical abundance, we have first corrected the spectra for foreground and internal reddening, using the Galactic reddening law as presented by Seaton (1979). First we have corrected the emission line fluxes (whose measurements are presented in Papers I and II) for the foreground reddening due to our Galaxy (see Table 1 of Kinney et al. 1993). Then we have

TABLE 1

Calculated Properties of the Star-Forming Galaxies

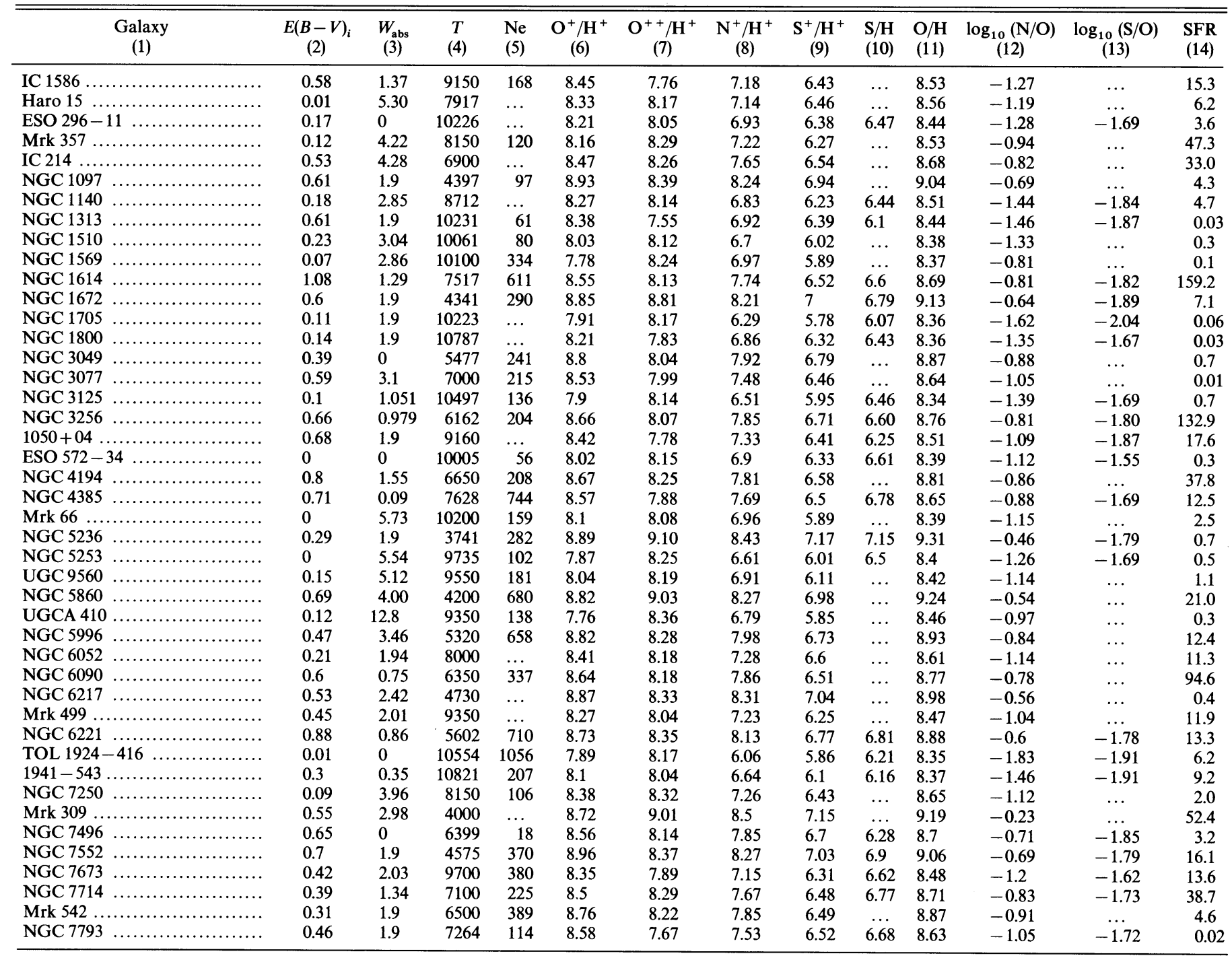


used the Balmer decrement in emission $\mathrm{H} \alpha / \mathrm{H} \beta$ to calculate the internal reddening for each galaxy assuming an intrinsic decrement of 2.9. As shown by McCall, Rybski, \& Shields (1985), the underlying stellar absorption features lead to a steepening of the Balmer decrement in emission and an overestimation of the extinction. In order to correct for this effect, they have used the following method: where $\mathrm{H} \gamma$ is detected in emission, the Balmer decrement is corrected simultaneously for the internal dust extinction and underlying stellar absorption; where it is not detected, an average underlying absorption of $1.9 \AA$ is assumed. Although McCall's method should be reliable for the determination of the reddening for the BCG, BCDG, and $\mathrm{H}$ II galaxies, it is not obvious it should apply to the starbursts of our sample, which show signatures of previous (and redder) generations of stars. We have then compared the reddening values $E(B-V)_{i}$ obtained using McCall's method with those obtained through an alternative method of taking into account the underlying absorption: we have selected in the library of Bica (1988) a stellar population template matching the equivalent widths $(W)$ of the absorption lines (the ones free from emission) of the observed spectra. Then, for each template, we have calculated the $W$ 's of $\mathbf{H} \alpha, \mathrm{H} \beta$, and $\mathrm{H} \gamma$ and have corrected the emission lines accordingly. The typical differences between the values of $E(B-V)_{i}$ obtained using this method and that of $\mathrm{McC}$ all are about $0.05 \mathrm{mag}$, which are within our uncertainties, except for NGC 1313 and IC 1586, where the differences are about $0.2 \mathrm{mag}$. As this difference for these galaxies does not affect our conclusions, and considering that the identification of the appropriate template was not obvious in some cases, we decided to adopt the $E(B-V)_{i}$ values obtained using McCall's method for all the spectra. The resulting internal reddening and equivalent width of underlying absorption are listed, respectively, in the second and third columns of Table 1.

\subsection{Temperature and Density}

In order to calculate the ionic abundances of $\mathrm{O}^{+}, \mathrm{O}^{++}, \mathrm{N}^{+}$, $\mathrm{S}^{+}$, and $\mathrm{S}^{++}$, we have used the three-level atom formulae presented by McCall (1984). These formulae provide the dependence of the emission coefficients not only on the temperature, but also on the electron density, thus accounting for the contribution of collisional excitation in the calculation of the abundances. As the resulting abundance values are very sensitive to the gas temperature (e.g., Garnett 1992), it is important to take into account the ionization structure of the nebulae. We have used a two-zone model (e.g., Campbell, Terlevich, \& Melnick $1986)$, in which the [O III] $\lambda \lambda 4959,5007 /[\mathrm{O}$ III $] \lambda 4363$ emissionline ratio gives the temperature corresponding to the zone of the high-ionization species, like $\mathrm{O}^{++}$and $\mathrm{Ne}^{++}$[which we hereafter refer to as $T(\mathrm{O}$ III)]. For the lower ionization species-like $\mathrm{O}^{+}, \mathrm{N}^{+}$, and $\mathrm{S}^{+}$, the temperature could be deter-

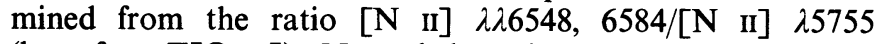
(hereafter $T\left[\begin{array}{ll}\mathrm{O} & \mathrm{II}\end{array}\right]$ ). Nevertheless, it was not possible to measure $[\mathrm{N}$ II $] \lambda 5755$ in any of our spectra, and we were able to measure [O III] $\lambda 4363$ in only a few, as these emission lines are usually very faint. We thus have used the calibration diagrams of Pagel et al. (1979) and Dopita \& Evans (1986; for the temperature range $7000<T[\mathrm{O} \mathrm{III}]<9000 \mathrm{~K}$ ) to obtain $T[\mathrm{O} \mathrm{III}]$ which is listed in the fourth column of Table 1.

$T[\mathrm{O}$ III $]$ was used in the calculations of the abundances of the ions $\mathrm{O}^{++}$and $\mathrm{Ne}^{++}$. Although previous authors have used $T[\mathrm{O} \mathrm{III}]$ as the temperature corresponding also to the zone producing $\mathrm{S}^{++}$, Garnett (1992) pointed out that the ionization structure of the nebula is such that the zone corre- sponding to $\mathrm{S}^{++}$is located between those producing $\mathrm{O}^{++}$and $\mathrm{O}^{+}$, and thus has an intermediate temperature. Using photoionization model calculations, he proposes a calibration of $T[\mathrm{~S}$ III $]$ as a function of $T[\mathrm{O} \mathrm{III}]$ which we have then used in the calculation of the $\mathrm{S}^{++}$abundance. $T[\mathrm{O} \mathrm{II}]$ was obtained from its parameterization as a function of $T[\mathrm{O} \mathrm{III}]$ as given by Campbell et al. (1986).

The gas density was determined from the [S II $] \lambda 6717 /[\mathrm{S}$ II $]$ $\lambda 6731$ ratio, using the relations given by McCall (1984) and $T[\mathrm{O}$ II $]$ as the temperature for the zone producing $\mathrm{S}^{+}$. The obtained values are listed in the fifth column of Table 1.

\subsection{Oxygen, Nitrogen, and Sulfur Abundances}

Using the above temperature and density values, we have then calculated the ionic abundances listed in columns (6)-(9) of Table 1. From the uncertainties on the measurements of the emission lines (Storchi-Bergmann et al. 1994) the estimated uncertainties on the abundance values are about 0.15 dex. From the ionic abundances, the total abundances relative to solar were then calculated. The oxygen was assumed to be present in the nebulae in essentially two ionization stages- $-\mathrm{O}^{+}$ and $\mathrm{O}^{++}$-and thus the total oxygen abundance is the sum of the abundances of these two ions. Its value is given in column 11 of Table 1. An additional check on the uncertainties was made through the comparison between the abundances obtained for four galaxies for which we have both spectra from KPNO (Paper II) and CTIO (Paper I): the difference in the abundances obtained from the different spectra for the same galaxies agree within $0.1 \mathrm{dex}$ in three cases and $0.15 \mathrm{dex}$ in one case. We have also compared our calculated oxygen abundances with those obtained using the recent calibrations of McGaugh (1991): the difference between the two sets of values are within $0.15 \mathrm{dex}$, and thus of the order of our uncertainties, although McGaugh's values are on average 0.1 dex higher than the calculated ones.

For nitrogen, we present in column (12) of Table 1 the logarithm of the ratio between its abundance and that of oxygen. We assume that $\mathrm{N} / \mathrm{O}=\mathrm{N}^{+} / \mathrm{O}^{+}$, based upon the rough coincidences between the ionization potentials of the two ions (Peimbert \& Costero 1969, used also in Alloin et al. 1979) to obtain nitrogen abundances of $\mathbf{H}$ II regions in external galaxies. Nevertheless, we should point out that recent calculations by Garnett (1990) have shown that N/O can be underestimated by this assumption due to the contribution of the ion $\mathrm{N}^{++}$to the nitrogen abundance. But, as we do not have access to the infrared [N III] emission lines, we will use the above approximation for $\mathrm{N} / \mathrm{O}$, keeping in mind that its value may be about 20\% larger (Garnett 1990).

The sulfur should be present in the nebulae in at least three ionization stages: $\mathrm{S}^{+}, \mathrm{S}^{++}$, and $\mathrm{S}^{+++}$(e.g., Garnett 1992); although for the northern sample we have only lines from $\mathrm{S}^{+}$, for the southern sample we have also the [S III] $\lambda \lambda 9068,9532$ emission lines in the near-IR. In this last case, Garnett (1989) has shown that the ratio $\left(\mathrm{S}^{+}+\mathrm{S}^{++}\right) / \mathrm{S}$ can be calculated from photoionization models of $\mathbf{H}$ II regions, and can be parameterized by the relation proposed by Stasińska (1978) and French (1981):

$$
\frac{\mathrm{S}^{+}+\mathrm{S}^{++}}{\mathrm{S}}=\left[1-\left(1-\frac{\mathrm{O}^{+}}{\mathrm{O}}\right)^{\alpha}\right]^{1 / \alpha}
$$

for $\alpha=2.5$. We have then used this parameterization to obtain the ratio $\mathrm{S} / \mathrm{O}$ between the sulfur and oxygen abundances (for 
the southern sample only, as we do not have near-IR spectra for the northern sample) which are presented in the column (13) of Table 1.

We will adopt the following notation throughout this paper: for each ionic or atomic species, the ratio $X / \mathrm{H}($ e.g., $\mathrm{O} / \mathrm{H}$ ) represents the logarithm of the ratio between the abundances (by number) of the ion $X$ and atoms of hydrogen plus 12 [e.g., $\log (\mathrm{O} / \mathrm{H})+12]$; and the ratio between two species (e.g., N/O) represents the logarithm of the ratio between their abundances \{also by number $[$ e.g., $\log (\mathrm{N} / \mathrm{O})]$.

\section{DISCUSSION}

\subsection{Nitrogen versus Oxygen Abundance}

We have plotted $\mathrm{N} / \mathrm{O}$ against $\mathrm{O} / \mathrm{H}$ in Figure 1, where it can be seen that, for $\mathrm{O} / \mathrm{H}>8.4, \mathrm{~N} / \mathrm{O}$ increases linearly with $\mathrm{O} / \mathrm{H}$. A least squares fit to the points gives

$$
\mathrm{N} / \mathrm{O}=0.96 \times \mathrm{O} / \mathrm{H}-9.29
$$

A linear relationship like the above with unity slope is the exact behavior predicted by a simple model of galactic chemical evolution with instantaneous recycling, in which nitrogen is a product of secondary nucleosynthesis and oxygen a product of primary nucleosynthesis (Talbot \& Arnett 1974; Serrano \& Peimbert 1983; Pagel 1985). A similar result was obtained by McCall et al. (1985) in the study of the abundances of $\mathrm{H}$ II regions in external galaxies: for the highest metallicity $\mathrm{H}$ II regions, the nitrogen arises mainly from secondary nucleosynthesis, the primary production being negligible.

We also note that the highest $\mathrm{N} / \mathrm{O}$ ratios found for the starbursts $(\approx 4$ times solar) are of the order of the highest ratios found in previous works (Storchi-Bergmann \& Pastoriza 1989, 1990; Storchi-Bergmann, Wilson, \& Baldwin 1992) for the narrow-line regions of Seyfert 2's and LINERs. The present study shows that such high ratios are not a characteristic only

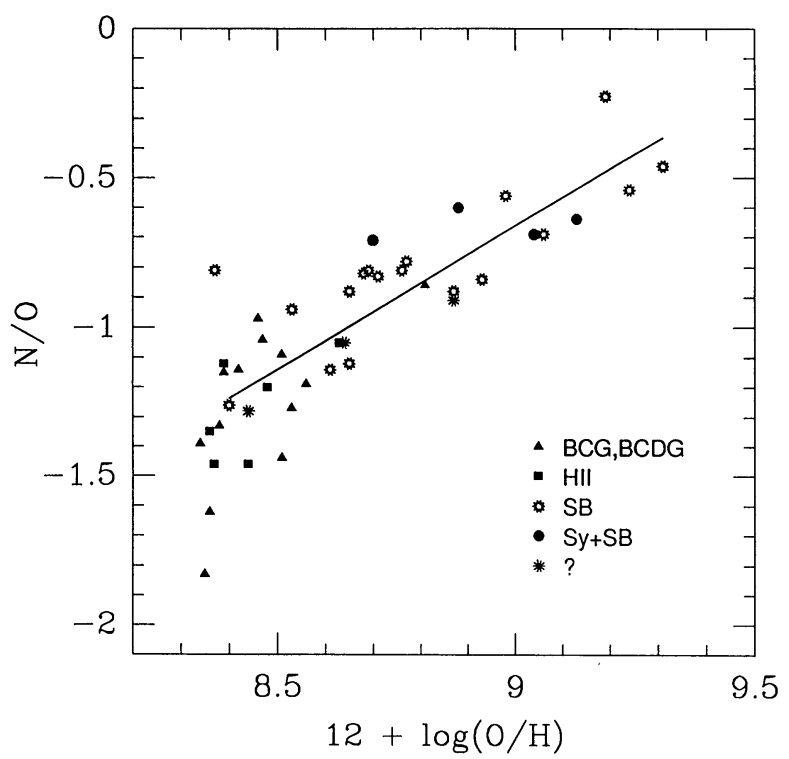

FIG. 1.-Logarithm of the number ratio of nitrogen to oxygen abundances plotted against $12+\log (\mathrm{O} / \mathrm{H})$. Triangles represent $\mathrm{BCG}$ or $\mathrm{BCDG}$; squares represent $\mathrm{H}$ II nuclei; starred symbols represent Starbursts; circles represent composite of Seyfert and Starbursts (but where the starburst characteristics are clearly dominant) and asterisks (*) represent the unclassified galaxies. The line is a least-squares fit to the data for $\mathrm{O} / \mathrm{H}>8.4$. of the narrow-line regions of active galaxies but are expected for galaxies which have experienced sufficient chemical processing, and are due to the secondary nature of nitrogen.

Other studies have shown that $\mathrm{N} / \mathrm{O}$ is uncorrelated with $\mathrm{O} / \mathrm{H}$ in dwarf galaxies (Garnett 1990, and references therein), where $\mathrm{O} / \mathrm{H}$ is low, leading to the suggestion that nitrogen is mostly a product of primary nucleosynthesis in low metallicity galaxies. But for higher metallicities there is an increasing contribution from secondary nucleosynthesis (Dinnerstein 1986; Garnett 1990). Our results are in agreement with these studies, as our galaxies can be considered a high-metallicity sample: the lowest metallicities are $\mathrm{O} / \mathrm{H} \approx 8.3$-which is about the largest metallicity of the sample of dwarf galaxies studied by Garnett (1990). The average value $\mathrm{N} / \mathrm{O}=-1.47$ found by Garnett for the dwarfs is also of the order of our lowest values.

Our findings thus support the conclusions of Matteucci (1986; also McCall et al. 1985) that primary nitrogen originate in high- and intermediate-mass stars, whereas secondary nitrogen would be confined to lower mass stars. Primary production would thus predominate at low and intermediate metallicities, and secondary production would dominate during the latest evolutionary phases, in galaxies which have experienced several bursts of star formation. Indeed, the highest metallicities - and highest $\mathrm{N} / \mathrm{O}$ ratios-are found in the galaxies for which we conclude that continuous bursts of star formation are occurring (see following sections).

We have plotted in Figure 2, $\mathrm{O} / \mathrm{H}$ against the emission-line ratio $[\mathrm{N} \mathrm{II]} / \mathrm{H} \alpha$. It can be seen that this ratio is very well correlated with the oxygen abundance for the range covered by our sample, namely $8.3<\mathrm{O} / \mathrm{H}<9.4$, with a Spearman correlation coefficient of $r_{s}=0.87$. A linear least-squares fit to the data gives the following relation (represented by a line in the figure):

$$
\mathrm{O} / \mathrm{H}=(0.91 \pm 0.09) \times[\mathrm{N} \mathrm{II}] / \mathrm{H} \alpha+(8.31 \pm 0.04) .
$$

As $[\mathrm{N}$ II $] / \mathrm{H} \alpha$ is not affected by reddening, and is easily observed both in the optical range for the nearby galaxies and in the near-IR for galaxies with a moderate redshift, the above

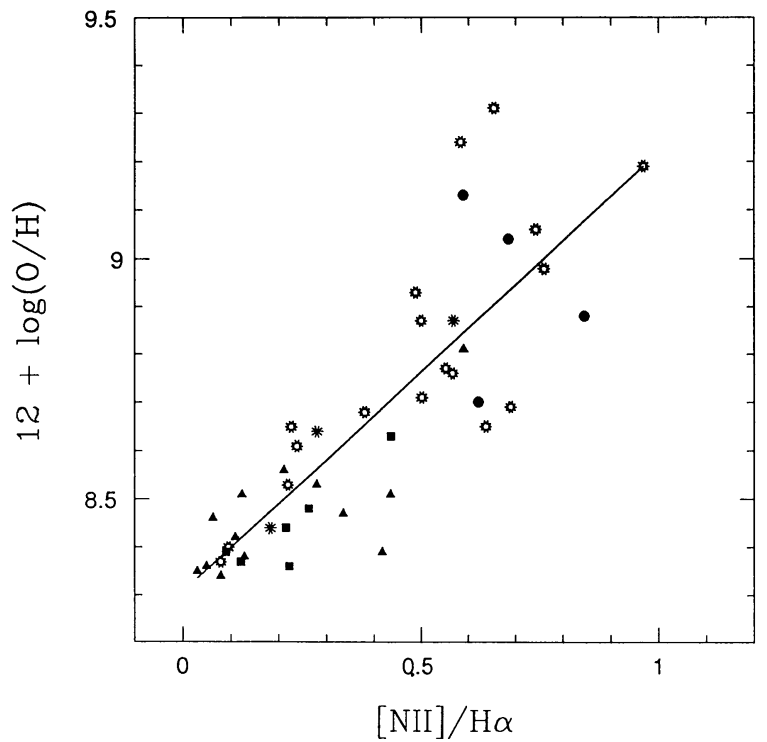

Fig. 2.-Oxygen abundance $12+\log (\mathrm{O} / \mathrm{H})$ plotted against the emission line ratio [N II] $\lambda \lambda 6548,6584 / \mathrm{H} \alpha$. Symbols are as in Fig. 1. The line shows a least-squares fit to the data. 
relation can be used to obtain the oxygen abundance of starforming galaxies with oxygen abundances in the range covered by our sample.

\subsection{Sulfur versus Oxygen Abundance}

We show in Figure 3 the results obtained for the $\mathrm{S} / \mathrm{O}$ ratio as a function of $\mathbf{O} / \mathbf{H}$. It can be seen that the scatter is large and that we do not find any correlation between the two quantities. Our results can be compared with those from Garnett (1990): although the $\mathrm{S} / \mathrm{O}$ ratios and their scatter are similar at low metallicities, at high metallicities our $\mathrm{S} / \mathrm{O}$ ratios are somewhat lower than those of Garnett (1990). Our data thus do not support the result of simple chemical evolution models as presented by Garnett (1989) which predict an increase of S/O with $\mathrm{O} / \mathrm{H}$ for the higher abundance objects. Nevertheless, as can be seen from Figure 3, the number of high-metallicity galaxies for which we have [S III] data is small; data on more galaxies are required to reach a firm conclusion.

\section{3. $U V$ Color versus $\mathrm{O} / \mathrm{H}$}

The continuum distribution of stellar populations is dependent on metallicity, reddening and age, as can be seen, for example, in the optical spectra of star clusters (e.g., Bica \& Alloin 1986a, b, and references therein).

In order to verify the effect of the metallicity on the continuum shape of the star-forming galaxies, we have studied the dependence of the color $C(14-35)=-25 \log \left(F_{\lambda 1355} / F_{\lambda 3500}\right)$ (Paper I) on $\mathrm{O} / \mathrm{H}$. Figure 4 shows that there is a correlation between these two quantities with $r_{s}=0.62$. A linear leastsquares fit to the data gives

$$
C(14-35)=(1.68 \pm 0.33) \times(\mathrm{O} / \mathrm{H})-(15.59 \pm 2.94) \text {. }
$$

In principle this relation could be very useful in determining the metallicity of faint distant galaxies, for which only continuum colors can be measured. Nevertheless, the scatter is very large, probably due to the effects of different reddenings and ages, and the relation can only give a very rough estimate of the metallicity.

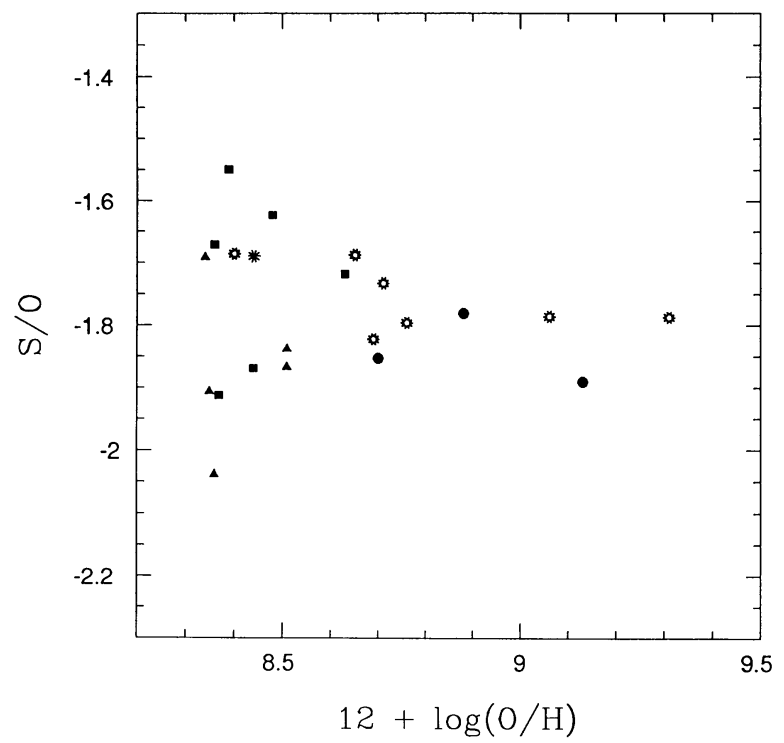

Fig. 3.-Logarithm of the number ratio of sulfur to oxygen abundance plotted against $12+\log (\mathrm{O} / \mathrm{H})$ (southern sample only). Symbols are as in Fig. 1.

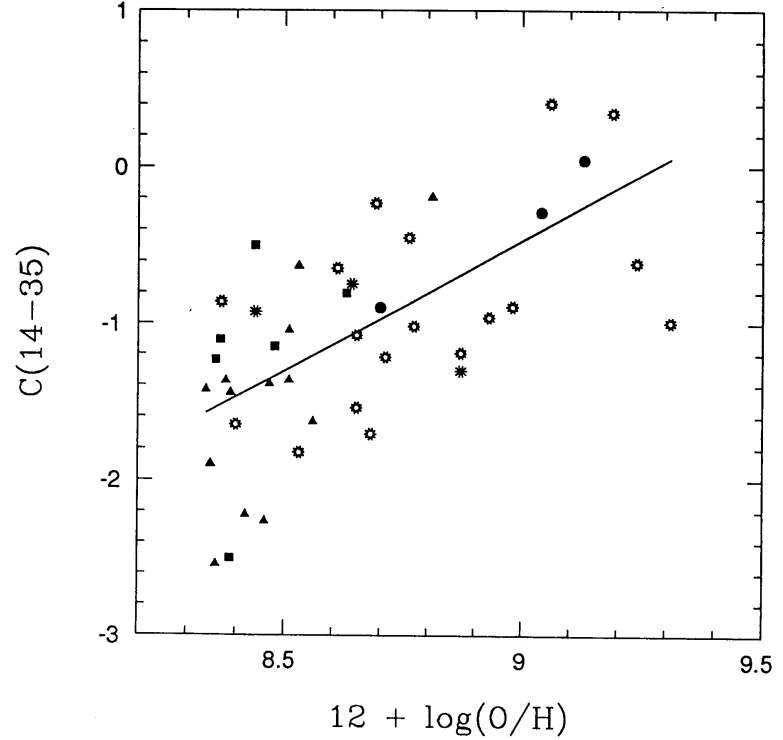

FIG. 4.-Continuum color $C(14-35)$ plotted against $12+\log (\mathrm{O} / \mathrm{H})$ Symbols are as in Fig. 1. The line is a least-squares fit to the data.

Calzetti et al. (1994) have investigated the effects of dust on the UV slope for the same sample studied here and found that the UV slope is primarily sensitive to the reddening. We have then plotted in Figure $5 a C(14-35)$ (after correction for the foreground Milky Way reddening) against $E(B-V)_{i}$. The correlation coefficient is now 0.77 , meaning that the continuum color is better correlated with $E(B-V)$ than with $\mathrm{O} / \mathrm{H}$, in agreement with Calzetti et al. (1994) results.

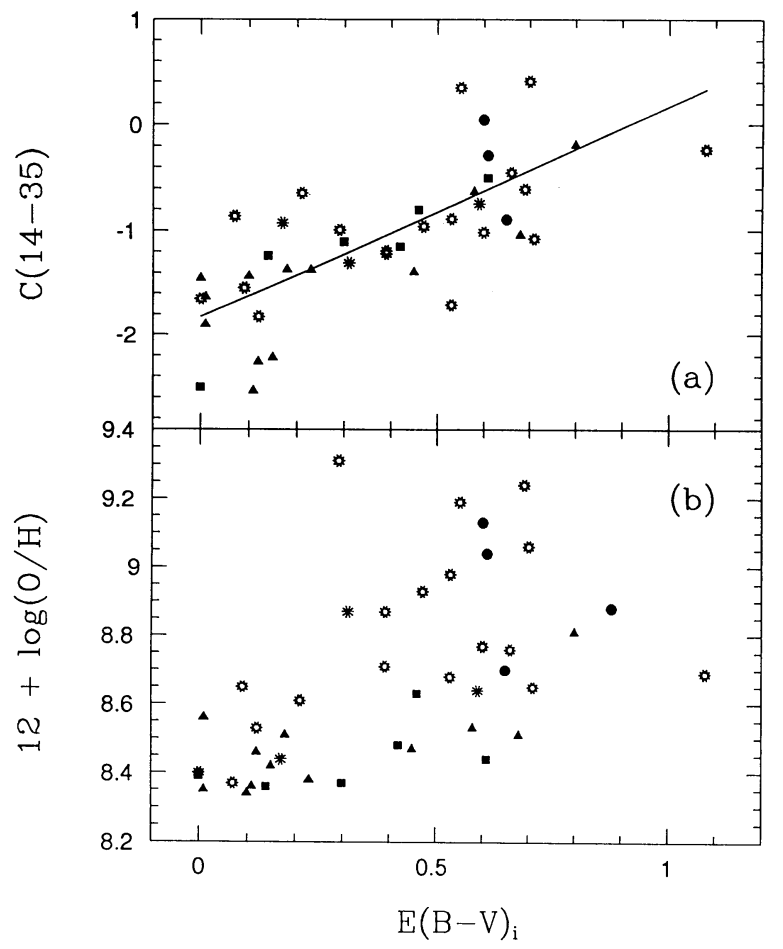

Fig. 5.-(a) Continuum color $C(14-35)$ plotted against the internal reddening $E(B-V)_{i} ;(b)$ oxygen abundance $12+\log (\mathrm{O} / \mathrm{H})$ plotted against $E(B-V)_{i}$. Symbols are as in Fig. 1. 
A least-square fit to the points gives

$$
C(14-35)=(1.99 \pm 0.28) \times E(B-V)_{i}-(1.82 \pm 0.13) \text {. }
$$

This relation suggests that, in the absence of reddening, the intrinsic average color of the galaxies is $C(14-35)=$ $-1.82 \pm 0.13$

In Figure $5 b$ we investigate the relation between $\mathrm{O} / \mathrm{H}$ and $E(B-V)_{i}$. Although the scatter is large, there is some correlation, with $r_{s}=0.65$. This correlation is expected, because if the galaxy is more metal-rich, the dust content (and reddening) will be higher (Lequeux 1988; Pei 1992). Nevertheless the correlation is not very significant, indicating that other factors besides metallicity determine the value of $E(B-V)_{i}$, such as the geometric distribution of the dust (Witt, Thronson, \& Capuano 1992; Calzetti et al. 1994) or the inclination of the galaxy relative to the line of sight (Bica \& Alloin 1986b).

Particularly noticeable is the position occupied by the galaxy NGC 5236, which is the one with higher $\mathrm{O} / \mathrm{H}(9.31)$ in our sample, but presents a relatively small $E(B-V)$. This galaxy is thus suitable for disentangling the effects of reddening and metallicity which we address in a separate paper (Kinney et al. 1994).

In an attempt to separate the effect of metallicity, we have used the galactic law (Seaton 1979) to correct $C(14-35)$ for reddening using the above $E(B-V)_{i}$. But when this corrected color is plotted against $E(B-V)_{i}$ the result is an inverse correlation! We have next tried to use the LMC reddening law (Fitzpatrick 1986) to correct the galaxies with $\mathrm{O} / \mathrm{H}<8.63$ (LMC-like; see Calzetti et al. 1994) and the galactic law for the galaxies with $\mathrm{O} / \mathrm{H}>8.63$. But the result is an even stronger inverse correlation for the lower metallicity galaxies, because the reddening-law for the LMC is steeper than the galactic law in the UV. These results indicate that the reddening correction has been overestimated, and the extinction affecting the UV continuum $E(B-V)_{\mathrm{UV}}$ is lower than that affecting the gas, a

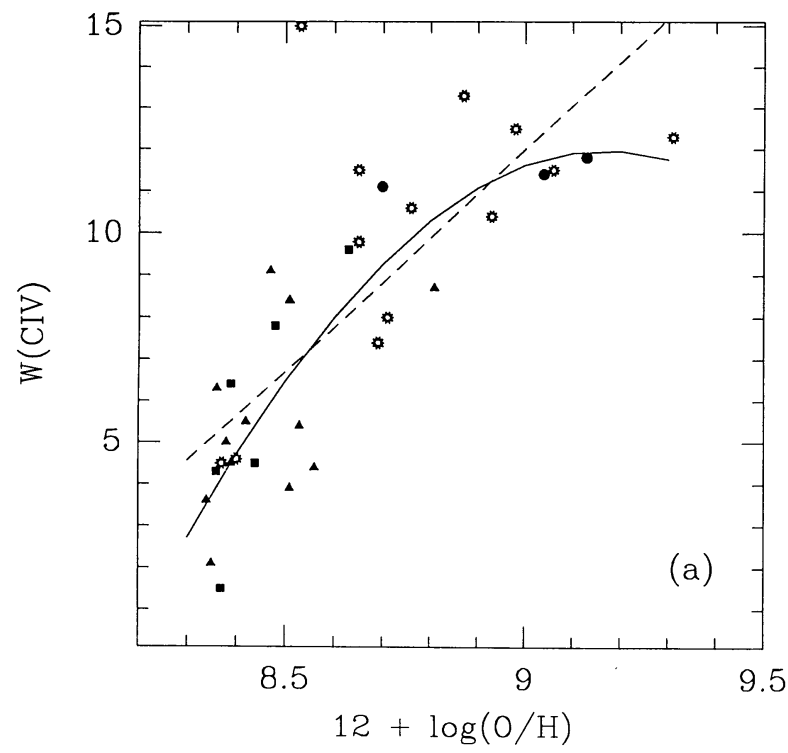

Fig. 6. - $($ a) Equivalent width $W(C$ IV $\lambda 1550)$ plotted against the oxygen abundance $12+\log (\mathrm{O} / \mathrm{H})$. Symbols are as in Fig. 1. Dashed line is a linear least squares fit to the data, and the continuous curve is a second order polynomium fit. $(b, c)$ Equivalent widths $W(\mathrm{Si}$ IV 21400$)$ and $W(\mathrm{Ca}$ IR) (see text) plotted against the oxygen abundance $12+\log (\mathrm{O} / \mathrm{H})$. result already pointed out by Fanelli et al. (1988). Fanelli et al. argue that the UV light preferentially emerges through windows of low optical depth, where the dust has been destroyed through the action of winds and ionizing radiation of the young stars. Nevertheless, the correlation between $C(14$ $35)$ and $E(B-V)_{i}$ argues for a relation between $E(B-V)_{\mathrm{Uv}}$ and $E(B-V)_{i}$. If we assume that $E(B-V)_{\mathrm{UV}}$ is proportional to $E(B-V)_{i}$, and that the intrinsic $E(B-V)_{\mathrm{UV}}$ would result in no correlation (positive or negative) between $C(14-35)$ and $E(B-V)_{i}$, we derive $E(B-V)_{\mathrm{Uv}}=0.45 \pm 0.10 \times E(B-V)_{i}$. As an exercise, we have then "corrected" $C(14-35)$ by this $E(B-V)_{\mathrm{UV}}$, and looked for a correlation between $C(14-35)$ and $\mathrm{O} / \mathrm{H}$; no correlation was found.

We conclude that it is not possible to separate the metallicity from the reddening effect, except perhaps for a few individual cases (e.g., NGC 5236, as pointed out above).

\subsection{Absorption-Line $W$ 's versus $\mathrm{O} / \mathrm{H}$}

In Paper I we presented the equivalent widths $W$ of a number of absorption features. Here we investigate the correlations between the different $W$ 's and $\mathrm{O} / \mathrm{H}$. In Figure 6 we show the corresponding values plotted against $\mathrm{O} / \mathrm{H}$ for the three cases where significant correlation was found. Typical uncertainties on the $W$ 's are about $1 \AA$.

$W(\mathrm{C}$ Iv $)(\lambda 1550)$ is very well correlated with $\mathrm{O} / \mathrm{H}$, with $r_{s}=$ 0.81 . Notice the large dynamical range presented by $W(\mathrm{C}$ IV $)$, which varies from 2 to $15 \AA$. A polynomial fit to the data gives

$$
\begin{aligned}
W(\mathrm{C} \text { IV })= & -(12.2 \pm 4.3) \times(\mathrm{O} / \mathrm{H})^{2}+(224 \pm 74) \\
& \times(\mathrm{O} / \mathrm{H})-(1017 \pm 324),
\end{aligned}
$$

while a linear fit gives

$$
W(\mathrm{C} \text { IV })=(10.6 \pm 1.3) \times(\mathrm{O} / \mathrm{H})-(83.4 \pm 10.8) .
$$

Both fits are shown in Figure $6 a$. Remarkably, the correlation

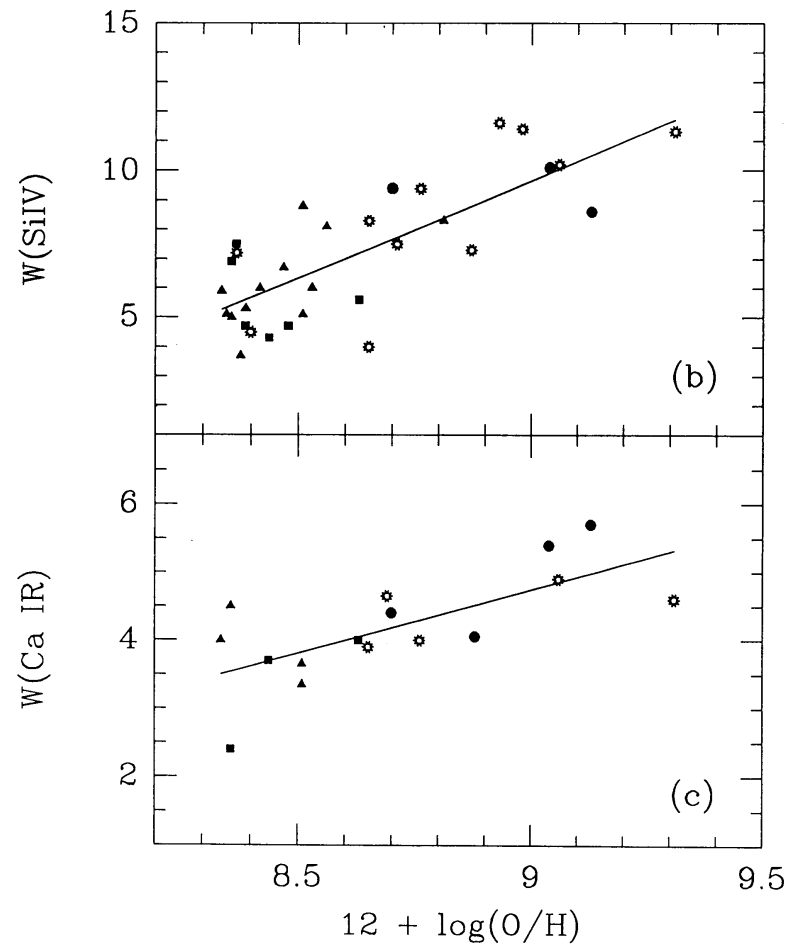


is highly significant, indicating that the metallicity is the dominant parameter in the determination of the $Q(\mathrm{C}$ IV) strength, and thus making the $\mathrm{C}$ IV absorption feature a good measure of the metallicity of a star-forming galaxy.

Figure $6 b$ shows that $W(\mathrm{Si}$ v) is also well correlated with $(\mathrm{O} / \mathrm{H})$. The correlation coefficient is $r_{s}=0.70$. A linear leastsquares fit to the data gives

$$
W(\mathrm{Si} \text { IV })=(6.6 \pm 1.0) \times \mathrm{O} / \mathrm{H}-(50.1 \pm 8.5) .
$$

$W(\mathrm{~S}$ IV) can thus also be used to measure the metallicity of a starburst from its UV spectrum.

The correlations of $W(\mathrm{C}$ IV) and $W(\mathrm{Si}$ IV) versus $\mathrm{O} / \mathrm{H}$ clearly show, for the first time, that the stellar metallicity is well correlated with the gas metallicity in star-forming galaxies, and can be understood as due to the fact that $\mathrm{C}$ IV and Si IV form only in hot stars, which are therefore young and have close to ISM abundances.

A good correlation was also found between $\mathrm{O} / \mathrm{H}$ and the $\mathrm{Ca}$ II triplet in the near IR, although for a smaller sample since the northern galaxies are not yet observed in the mean IR, and the southern galaxies were not all detected (see Paper I). In Figure $6 c$ we have plotted the average between $\mathrm{Ca}$ II $\lambda 8542$ and $\mathrm{Ca}$ II $\lambda 8662$, which we have called $W(\mathrm{CaIR})$. The correlation coefficient is $r_{s}=0.75$. A linear least-squares fit gives

$$
W(\mathrm{CaIR})=(1.87 \pm 0.50) \times(\mathrm{O} / \mathrm{H})-(12.08 \pm 4.37) \text {. }
$$

$W(\mathrm{CaIR})$ could thus be another metallicity indicator for the star-forming galaxies. Nevertheless, we notice that its dynamic range is very small, and thus the above relation is only useful for very good $\mathrm{S} / \mathrm{N}$ ratio data. This metallicity dependence is probably due to the fact that the main contributors to these lines are giants and supergiants (Jones, Alloin, \& Jones 1984) which have recently evolved from the main sequence and thus show their metallicity correlated to that of the gas.

Little correlation was found between $W(\mathbf{C a}$ II $\mathrm{K})$, $W(\mathrm{Mg} \mathrm{I}+\mathrm{MgH})$ and $\mathrm{O} / \mathrm{H}$ (correlation coefficients of 0.54 and 0.42 , respectively). This is due to the fact that these features, which are easily observed in red stars, become diluted by the continuum from the young blue stars (Bica \& Alloin 1986a). Different proportions of young and old stellar population contributions will lead to different dilution in the absorption lines and thus will introduce scatter in the correlations.

We also did not find any correlation between $W(\mathrm{Fe} \mathrm{II})$, $W(\mathrm{Mg}$ II), and $\mathrm{O} / \mathrm{H}$, probably due to the interstellar contribution from the Milky Way and/or from the galaxy itself to these lines (Kinney et al. 1993). Their equivalent widths could depend on the amount and distribution of the interstellar matter and probably on the inclination of the galaxy as well, as found by Bica \& Alloin (1986b) for $\mathrm{Na}$ I 25890 . We have looked for a similar effect in our sample, investigating a possible correlation between $W\left(\mathrm{Fe}\right.$ II), $W(\mathrm{Mg}$ II $)$ and $E(B-V)_{i}$. We show in Figure $7 W(\mathrm{Mg}$ II) versus $E(B-V)$. Very little correlation is found $\left(r_{s}=0.44\right)$, although we have in this plot a reduced number of objects due to the lack of the LWP IUE spectra for a large fraction of the sample galaxies.

\subsection{Absolute Magnitude and Star-Formation Rate}

It is a well known fact that in early type galaxies, when the absolute luminosity increases, there is a strengthening of absorption lines and a reddening of the continuum which is attributed to an increasing metallicity (Faber 1977; Sandage \& Visvanathan 1978; Bica \& Alloin 1987). In order to verify if such an effect is also present for the starbursts, we have plotted

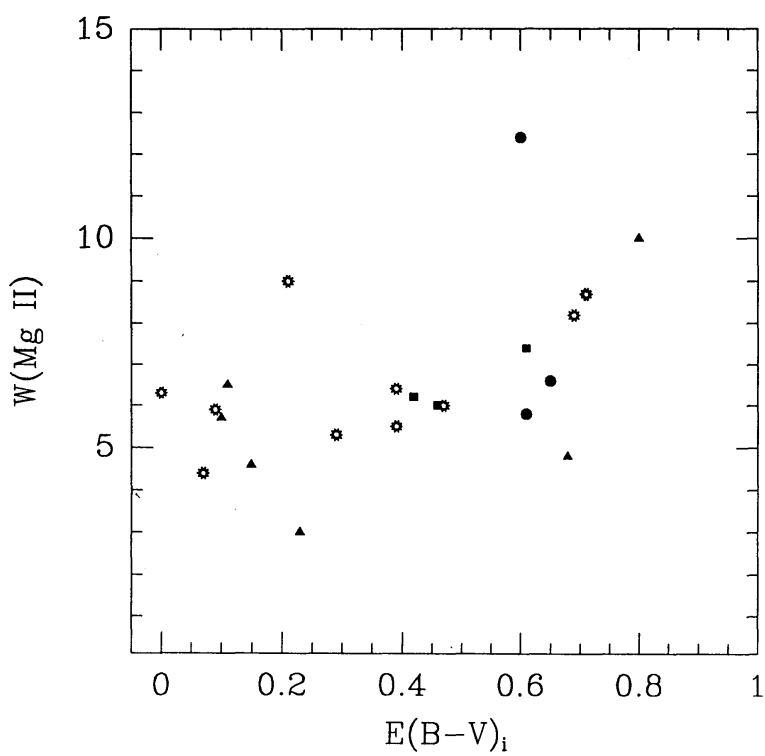

FIG. 7.-Equivalent width $W(\mathrm{Mg}$ II 22800$)$ plotted against internal reddening $E(B-V)_{i}$

in Figure 8 the absolute magnitude $M_{B}$ (Kinney et al. 1993) against $\mathrm{O} / \mathrm{H}$. The plot shows that the higher abundance galaxies are always luminous and the less luminous galaxies tend to be more metal poor, in agreement with previous results. But we also find that there is not a tight correlation between the two quantities, as the more metal poor galaxies span the whole $M_{B}$ range, from dwarfs to the more luminous galaxies. Inspection of the morphological types (Kinney et al. 1993) of the galaxies reveals that the more metal-rich starbursts are found in the more "early-type" galaxies, usually Sb's or Sc's, while the more metal poor are found in the ones classified as Sm, Irregular, or Amorphous. We thus interpret our results as being due to the fact that the metal-rich starbursts occur in evolved luminous galaxies that have experienced previous bursts of star-formation, which have enriched the gas from

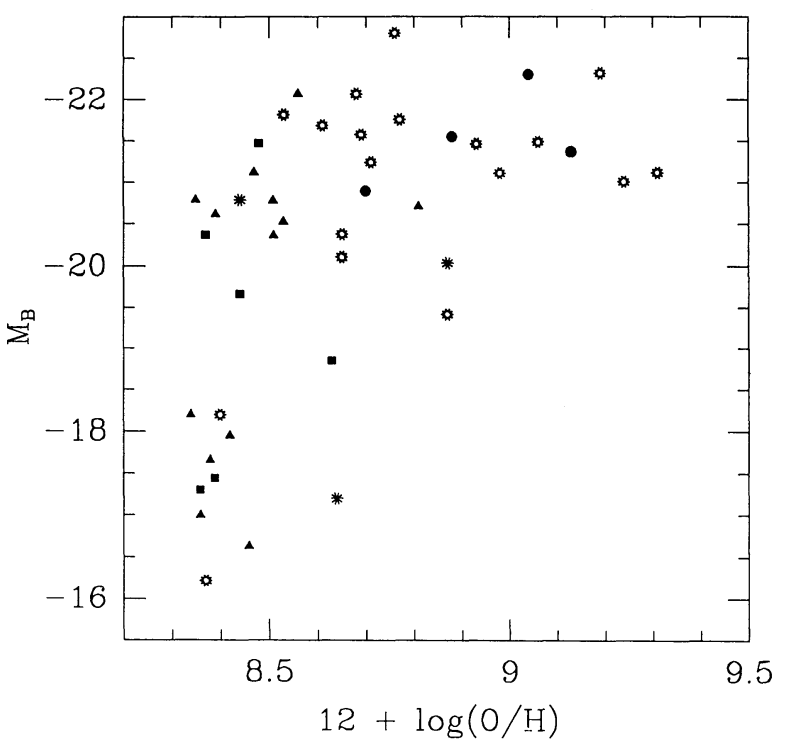

FIG. 8.-Absolute blue magnitude $M_{B}$ plotted against the oxygen abundance $12+\log (\mathrm{O} / \mathrm{H})$ 
which the new stars are forming. The more metal-poor ones are less evolved, havng been formed probably more recently, and the different luminosities are due to the different sizes of the protogalactic clouds. The more luminous bursts will originate in the more massive galaxies and the less luminous bursts will originate in the less massive galaxies. We indeed find a correlation between the $\mathrm{H} \alpha$ luminosity and the galaxy luminosity, as described below.

The $\mathrm{H} \alpha$ luminosity was calculated using the fluxes listed in Papers I and II after correction for reddening and using the distances from Kinney et al. (1993: $H_{0}=50 \mathrm{~km} \mathrm{~s}^{-1} \mathrm{Mpc}^{-1}$ ). From the $\mathrm{H} \alpha$ luminosity, we have then calculated the stellar formation rate SFR-which is proportional to the $\mathrm{H} \alpha$ luminosity-according to Kennicutt (1983). The corresponding values, in units of solar masses per year are listed in the last column of Table 1 . We have then looked for correlations between the SFR and $C(14-35), \mathrm{O} / \mathrm{H}$ and absolute magnitude $M_{B}$. Figure 9 shows that there is a good correlation between the decimal logarithm of the SFR and $M_{B}$, with $r_{s}=0.78$. A linear least-squares fit to the data gives

$$
\log _{10}(\mathrm{SFR})=(-0.49 \pm 0.06) \times M_{B}-(9.52 \pm 1.13)
$$

For distant blue galaxies, the SFR can be obtained from the equivalent width of [O II] $\lambda 3727$ (Kennicut 1992; Gallagher, Bushouse, \& Hunter 1989), and the above relation could thus be used to get a rough estimate of $M_{B}$, to be compared with the value derived through the measure of the radial velocity. Thus, in principle, this relation could be useful to measure deviations from the Hubble flow.

The SFR is not correlated with $\mathrm{O} / \mathrm{H}$ : for low metallicities, a whole range of SFR values is observed, from 0.01 to $100 M_{\odot}$ $\mathrm{yr}^{-1}$, while for the more metal-rich, only SFR larger than 1 $M_{\odot} \mathrm{yr}^{-1}$ are observed. This behavior is identical to that observed for $\mathbf{M}_{B}$, and results from the fact that SFR and $M_{B}$ are linearly correlated.

The above results thus show that the larger starbursts are found in the more luminous galaxies and that there is no correlation between the star formation rate and the metallicity of

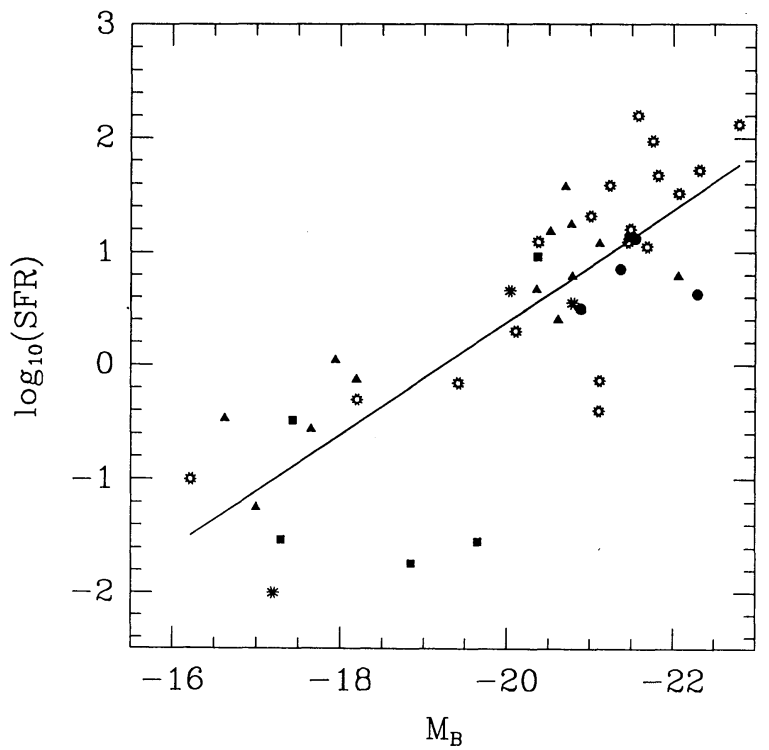

Fig. 9.-Decimal logarithm of the star-formation rate plotted against the absolute blue magnitude $M_{B}$. the gas. There is also no correlation between SFR (or size of the burst) and the continuum color $C(14-35)$.

\subsection{Age Effects}

Copetti, Pastoriza, \& Dottori (1986) studied the effects of the aging of a burst of star formation on the surrounding $\mathrm{H}$ III regions. They have demonstrated that, if a single burst is considered, $W(\mathbf{H} \beta)$ and $[\mathrm{O} \mathrm{III}] / \mathbf{H} \beta$ are good age indicators, both being large for a very young burst and decreasing as the burst ages. Nevertheless, for $W(\mathbf{H} \beta)$, the presence of an older generation of stars increases the continuum under $\mathrm{H} \beta$, and can lead to a small $W(\mathrm{H} \beta)$ even for a young burst. [O III] $/ \mathrm{H} \beta$ would be a better age indicator in these cases. But, on the other hand, this last ratio is very sensitive to the gas metallicity.

We have used the results of the work of Copetti et al. (1986) to look for an age effect in the spectral distributions of the star-forming galaxies. We have tried to separate the metallicity dividing the sample in three metallicity bins: the first with $\mathrm{O} / \mathrm{H} \leq 8.5$, the second with $8.5<\mathrm{O} / \mathrm{H}<8.7$ and the third with $\mathrm{O} / \mathrm{H} \geq 8.7$. Although the separation by metallicity is necessary, the resulting subsamples are small, so that strong conclusions cannot be drawn. In order to study the dependence of $C(14-35)$ on age, we have plotted in Figure 10, $C(14-35)$ versus $[\mathrm{O} \mathrm{III}] / \mathrm{H} \beta$ for the three metallicity bins above. We have also taken into account the reddening by representing with different symbols in the figures the objects with $E(B-V)<0.2,0.2 \leq E(B-V) \leq 0.5$ and $E(B-V)>0.5$. The figure shows that for the first (low-metallicity) bin, and low reddening there is some correlation $\left(r_{s}=0.74\right)$ which could be attributed to an age spread [we have excluded NGC 1569 because of its low Galactic latitude, and corresponding strong Galactic extinction (Kinney et al. 1993), which makes C(14-35)

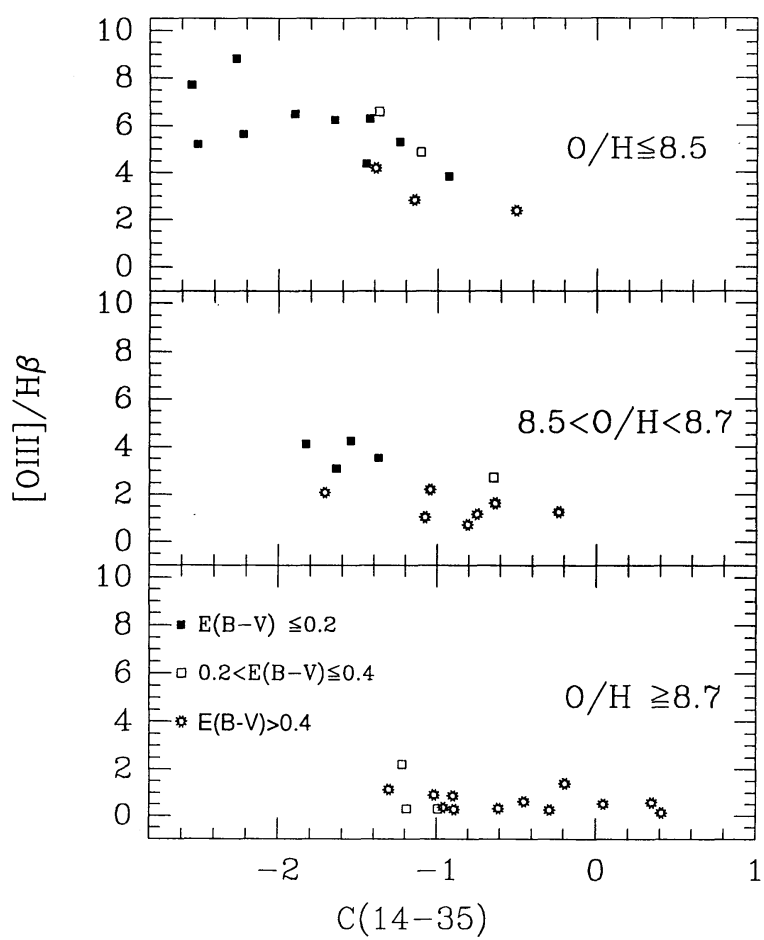

FIG. 10.-Emission line ratio [O III] $\lambda \lambda 4959,5007 / \mathrm{H} \beta$ plotted against the continuum color $C(14-35)$ for three metallicity bins. Different symbols are used to identify different reddenings. 
highly uncertain]. For the bin with $8.5 \leq \mathrm{O} / \mathrm{H} \leq 8.7$, the relation between $C(14-35)$ and [O III]/H $\beta$ seems to be dominated by a residual metallicity (and reddening) effect, and for the higher metallicity bin, [O III]/H $\beta$ almost does not vary, being always smaller than 2. From the models of Copetti et al. (1986), [O III] $/ \mathrm{H} \beta$ can be used to estimate that the lower metallicity galaxies have burst ages smaller than $3 \times 10^{6}$ yr. For the higher metallicity galaxies, the models show a very fast decrease of $[\mathrm{O} \mathrm{III}] / \mathrm{H} \beta$ with age, so that we can only estimate that the ages are larger than $2 \times 10^{6} \mathrm{yr}$. Alternatively, the hypothesis of a single burst (used in the models) may be not valid for the higher metallicity galaxies, which have experienced previous episodes of star formation.

One other method for estimating the age of star-forming galaxies was recently proposed by Robert, Leitherer, \& Heckman (1993): using stellar evolutionary models, they have shown that $W(\mathrm{Si}$ IV $\lambda 1400)$ and $W(\mathrm{C}$ IV $\lambda 1550)$, as well as the ratio between the two, show an age dependence in the case of an instantaneous burst of star formation. We have then compared our measurements with the model values and verify that, as pointed out already by Robert et al. (1993), the measured $W$ (Si Iv) values for the galaxies are somewhat larger than those from the models, while the $W(\mathrm{C}$ Iv) values are well within the range covered by the models. We have looked for relations between $C(14-35)$ and $W(\mathrm{Si} I V), W(\mathrm{C}$ IV) and found that, for the lower metallicity bin and lowest reddening, there is a trend for $W(\mathrm{Si}$ IV $) / W(\mathrm{C}$ IV) to increase as $C(14-35)$ gets redder (Fig. 11a), which is the predicted behavior for this ratio as the burst ages, due to the weakening of $W(\mathrm{C}$ IV) relative to $W(\mathrm{Si}$ IV $)$, as the number of $\mathrm{O}$ stars decreases (Robert et al. 1993; Wu et al. 1991). [There is one point deviating from the trend which cor-

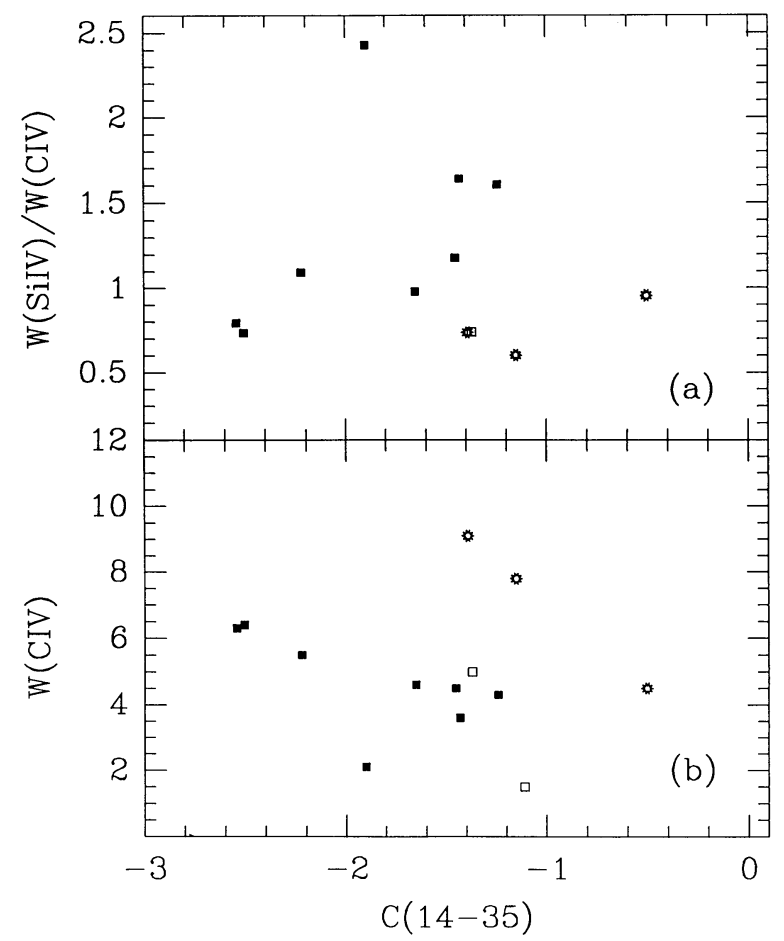

Fig. 11. - (a) The ratio between the equivalent widths $W($ Si IV $\lambda 1400)$ and $W(\mathrm{C}$ IV $\lambda 1550)$, and $(b)$ the equivalent width $W(\mathrm{C}$ IV $\lambda 1550)$ plotted against the continuum color $C(14-35)$ for the galaxies with $\mathrm{O} / \mathrm{H}<8$.5. Symbols are as in Fig. 9. responds to Tol 1924-416; this galaxy has a particularly strong emission in $\mathrm{C}$ IV in a $\mathbf{P}$ Cygni profile, which decreases the value of $W(\mathrm{C}$ IV $)$, as the emission is inside the window used to measure $W(\mathrm{C}$ IV $)$.]. Figure $11 b$ also shows a trend for a decreasing $W(\mathrm{C}$ IV $)$ as the color gets redder for the same galaxies. For the other metallicity bins, no correlation was found. It is interesting to point out that the same (low reddening and low metallicity) galaxies which present a correlation between [O III] $/ \mathrm{H} \beta$ and $C(14-35)$ are the ones showing the expected trend in the diagram $W(\mathrm{Si}$ Iv $) / W(\mathrm{C}$ Iv) versus $C(14-35)$, suggesting that we are indeed seeing an age effect in the continuum color for these galaxies. Comparison between the observed $W(\mathrm{C}$ IV) with the model predictions indicate ages in the range $5 \times 10^{6}-10^{7}$ yr, about 2 times larger than those obtained from [O III $] / \mathrm{H} \beta$. For the highest metallicity bin, the data shows approximately constant $W(\mathrm{Si} \quad$ IV $)=9.5 \pm 2$ and $W(\mathrm{C}$ IV $)=11 \pm 2$. This value of $W\left(\mathrm{C}_{\text {IV }}\right)$ is about the predicted one for a continuous burst of star formation, which supports the picture based on the observed $[\mathrm{O} \mathrm{III}] / \mathrm{H} \beta$ that these galaxies are not experiencing a single burst of star formation.

\section{SUMMARY AND CONCLUSIONS}

We have studied the effects of metallicity, star-formation rate and age in the UV to near-IR spectral distributions of 44 star-forming galaxies.

From the nebular emission lines, the gas abundances and stellar formation rate are calculated. The oxygen abundance is found to span the range $8.3<12+\log (\mathrm{O} / \mathrm{H})<9.4$, and the star-formation rate (SFR) ranges from 0.01 to $100 \mathrm{M}_{\odot} \mathrm{yr}^{-1}$.

We investigate correlations between spectral features and $\mathrm{O} / \mathrm{H}$ and find a highly significant correlation between the oxygen abundance $\mathrm{O} / \mathrm{H}$ and the absorption-line equivalent width $W(\mathrm{C}$ IV 21550$)$, indicating that this absorption feature is a good indicator of the stellar metallicity. Thus we show for the first time that the stellar metallicity is well correlated with the gas metallicity in star-bursting galaxies. We present a calibration which can be used to derive $\mathrm{O} / \mathrm{H}$ from the measurement of $W(\mathrm{C}$ IV $\lambda 1550)$. This result is of importance for observations with $H S T$ : from simply observing a limited UV wavelength range of a star-forming galaxy, the metallicity can be derived. The calibration presented here is valid for the abundance range covered by our sample $(8.3 \leq \mathrm{O} / \mathrm{H} \leq 9.3)$. It would be of great interest to use the Space Telescope to obtain good $\mathrm{S} / \mathrm{N}$ ratio UV spectra of more metal poor galaxies to extend the calibration to the lowest metallicity range.

We also find significant correlations between $\mathrm{O} / \mathrm{H}$ and the absorption-line equivalent width $W(\mathrm{Si}$ IV $\lambda 1400)$ and between $\mathrm{O} / \mathrm{H}$ and the emission-line ratio [N II] $\lambda \lambda 6548,84 / \mathrm{H} \alpha$, and we present calibrations that can be used to obtain $\mathrm{O} / \mathrm{H}$ from these two quantities in the abundance interval covered by this work.

The $\mathrm{N} / \mathrm{O}$ ratio scales linearly with $\mathrm{O} / \mathrm{H}$ for $12+\log$ $(\mathrm{O} / \mathrm{H})>8.4$, indicating that $\mathrm{N}$ is predominantly of secondary origin for galaxies with these metallicities. Due to its secondary origin, $\mathrm{N} / \mathrm{O}$ ratios up to $\approx 4$ times the solar value are obtained for the starbursts with highest $\mathrm{O} / \mathrm{H}$. We do not find any correlation between $\mathrm{S} / \mathrm{O}$ and $\mathrm{O} / \mathrm{H}$.

There is a correlation between the color $C(14-35)$ and $\mathrm{O} / \mathrm{H}$, although the spread is large. A more significant correlation is found between $C(14-35)$ and the internal reddening $E(B-V)_{i}$ confirming the results of Calzetti et al. (1994). We attempt to disentangle the metallicity from the reddening in the color $C(14-35)$ but do not succeed, as the metallicity is also correlated with $E(B-V)_{i}$. We verify that the reddening affecting the 
UV continuum is significantly smaller than that derived from the gas Balmer decrement, its value being estimated as $E(B-V)_{\mathrm{UV}}=0.45( \pm 0.10) \times E(B-V)_{i}$.

The larger metallicities are always found in the more luminous galaxies, while low metallicities can be found over all the luminosity range $\left(-16<M_{B}<-23\right)$. The star-formation rate (SFR) correlates well with the luminosity of the galaxy: the larger starbursts are found in the most luminous galaxies. The continuum color $C(14-35)$ does not depend on the size of the burst.

In order to investigate the dependence of $C(14-35)$ on the burst age, we use two age indicators for an instantaneous burst of star formation: $W(\mathrm{Si}$ IV $) / W(\mathrm{C}$ IV) from stellar synthesis models and $[\mathrm{O} \mathrm{III}] / \mathrm{H} \beta$ from models of the surrounding $\mathrm{H}$ II regions. Some dependence of $C(14-35)$ on these indicators is found only for the lowest metallicity galaxies $(\mathrm{O} / \mathrm{H} \leq 8.5)$. From the $\mathrm{H}$ II region models we derive ages smaller than
$3 \times 10^{6} \mathrm{yr}$ while from the stellar synthesis models we derive ages in the range 5-10 $\times 10^{6} \mathrm{yr}$. The highest metallicity galaxies have most likely experienced a continuous burst of star formation.

We acknowledge clarifying discussions with C. Robert, C. Leither, T. Heckman, A. R. Petrosian, D. Garnett, and E. Bica and thank the referee, F. Hamann, for useful suggestions. T. S. B. thanks the hospitality of STScI, where part of this work was done, and the Brazilian Institutions $\mathrm{CNPq}$, CAPES, and FAPERGS for partial support. D. C. and A. L. K. acknowledge support from the NASA grant NAG 5-1143 and from the STScI Directors Research Fund. A. L. K. would like to thank $\mathrm{CNPq}$ for support for a collaborative visit to the Departamento de Astronomia, IF-UFRGS.

Alloin, D., Collin-Souffrin, S., Joly, M., \& Vignoux, L. 1979, A\&A, 78, 200

Bica, E. 1988, A\&A, 195, 76

Bica, E., \& Alloin, D. 1986a, A\&A, 162, 21

1986b, A\&A, 166, 83 1987, A\&A, 181, 270

Bruzual, G. 1983, ApJ, 273, 105

Calzetti, D., Kinney, A. L., \& Storchi-Bergmann, T. 1994, ApJ, in press

Campbell, A., Terlevich, R. J., \& Melnick, J. 1986, MNRAS, 223, 811

Copetti, M. V. F., Pastoriza, M. G., \& Dottori, H. A. 1986, A\&A, 156, 111

Dinnerstein, H. L. 1986, PASP, 98, 979

Dopita, M. A., \& Evans, I. N. 1986, ApJ, 307, 431

Dressler, A., \& Gunn, J. E. 1983, ApJ, 270, 7

Dressler, A., Gunn, J. E., \& Schneider, D. P. 1985, ApJ, 294, 70

Ellis, R 1992 in IAU Symp. 149, The Stellar Population of Galaxies, ed. B. Barbuy \& A. Renzini (Dordrecht: Reidel), 297

Faber, S. 1977, in Evolution of Galaxies and Stellar Populations, ed. B. Tinsley \& R. Larson (New Haven: Yale Univ. Obs.), 157

Fanelli, M. N., O’Connell, R. W., \& Thuan, T. X. 1988, ApJ, 334, 665

Fitzpatrick, E. L. 1986, AJ, 92, 1068

French, H. B. 1981, ApJ, 246, 434

Gallagher, J. S., Bushouse, H., \& Hunter, D. A. 1989, AJ, 97, 700

Garnett, D. R. 1989, ApJ, 345, 282

$$
\text { 1990, ApJ, 363, } 142
$$

Guiderdoni, B., \& Rocca-Volmerange, B. 1987, A\&A, 186, 1

Hamman, F., \& Ferland, G. 1992, ApJ, 391, L53

Jones, J. E., Alloin, D. M., \& Jones, B. J. T. 1984, ApJ, 283, 457

Kennicutt, R. C., Jr. 1983, ApJ, 272, 54

1992, ApJ, 388, 310

Kinney, A. L., Bohlin, R. C., Caizetti, D., Panagia, N., \& Wyse, R. F. G. 1993, ApJS, 86, 5

Kinney, A. L., Calzetti, D., Bica, E., \& Storchi-Bergmann, T. 1994, ApJ, in press

Lequeux, J. 1988, in Dust in the Universe, ed. M. E. Bailey \& D. A. Williams

\section{REFERENCES}

Matteucci, F. 1986, PASP, 98, 973

McCall, M. L. 1984, MNRAS, 208, 253

McCall, M. L., Rybski, P. M., \& Shields, G. A. 1985, ApJS, 57, 1

McGaugh, S. S. 1991, ApJ, 380, 140

McQuade, K. A., Kinney, A. L., \& Calzetti, D. 1994, in preparation (Paper II)

Pagel, B. E. J. 1985, in Production and Distribution of CNO Elements, ed. I. J Danziger, F. Matteucci, \& K. Kjär (Garching: ESO), 155

Pagel, B. E. J., Edmunds, M. G., Blackwell, D. E., Chen, M. S., \& Smith, G. 1979, MNRAS, 189,95

Pei, Y.C. 1992, ApJ, 395, 130

Peimbert, M., \& Costero, R. 1969, Bol. Obs. Tonantzintla y Tacubaya, 5, 3

Robert, C., Leitherer, C., \& Heckman, T. 1993, ApJ, 418749

Sandage, A. 1986, A\&A, 161, 89

Sandage, A., \& Visvanathan, N. 1978, ApJ, 225, 742

Seaton, M. J. 1979, MNRAS, 187, 73P

Serrano, A., \& Peimbert, M. 1983, Rev. Mexicana Astron. Af., 8, 117

Stasińska, G. 1978, A\&A, 66, 257

Storchi-Bergmann, T., Kinney, A. L., Challis, P., \& Panagia, N. 1994, in preparation (Paper I)

Storchi-Bergmann, T., \& Pastoriza, M. G. 1989, ApJ, 347, 195

. 1990, MNRAS, 204, 347

Storchi-Bergmann, T., Wilson, A. S., \& Baldwin, J. A. 1992, ApJ, 396, 45

Talbot, R. J., \& Arnett, W. D. 1974, ApJ, 190, 605

Terlevich, R. J. 1990, in Windows on Galaxies, ed. G. Fabbiano, J. Gallagher \& A. Renzini (Dordrecht: Kluwer), 87

Terlevich, R. J., Tenorio-Tagle, G., Franco, J., \& Melnick, J. 1992, MNRAS 255,713

Tyson, J. A. 1984, PASP, 96, 566

1988, AJ,96, 1

Witt, A. N., Thronson, H. A., \& Capuano, J. M. 1992, ApJ, 393, 611

Wu, C.-C., Crenshaw, D. M., Blackwell, J. H., Jr., Wilson-Diaz, D., Schiffer, F. H. III, Burstein, D., Fanelli, M. N., \& O'Connell, R. W. 1991, IUE Newsletter no. 43

(Cambridge: Cambridge Univ. Press), 449 